\title{
FISIOTERAPIA E A SUA PRÁXIS NA ATENÇÃO BÁSICA \\ - um estudo sob a ótica dos discentes \\ e docentes da área de saúde em uma universidade pública na bahia•
}

\author{
Marcio Costa de Souza ${ }^{a}$ \\ Ângelo de Andrade Rocha ${ }^{b}$ \\ Jairrose Nascimento Souza ${ }^{c}$
}

\begin{abstract}
Resumo
O presente estudo teve como objetivo conhecer a percepção dos discentes e docentes da área de saúde de uma Universidade na Bahia sobre a inserção e a práxis do fisioterapeuta na Atenção Básica. Metodologia: apresenta uma abordagem qualitativa numa perspectiva histórico-social, no qual foram entrevistados dez indivíduos por meio de entrevista semiestruturada sendo divididos em dois grupos, dos quais, o grupo I foi composto por discentes e o grupo II por docentes. Os dados foram produzidos a partir da análise de conteúdo. Segundo os participantes da pesquisa é necessário a presença do fisioterapeuta na estratégia de saúde da família, porém percebe-se uma indefinição do objeto de trabalho deste profissional, principalmente quando necessita de ação interdisciplinar, além de culturamente ser estabelecida uma práxis reabilitadora. Desta forma, é importante que haja uma mudança imediata na formação profissional do fisioterapeuta, uma maior divulgação sobre o papel e a importância desse profissional na Atenção Básica além de pesquisas na área que promovam esse debate que envolva a necessidade do usuário.
\end{abstract}

Palavras-chave: Atenção Primária à Saúde; Fisioterapia; Recursos Humanos.

\footnotetext{
a. Fisioterapeuta. Mestre em Saúde coletiva, Docente da Universidade do Estado da Bahia. mcsouzafisio@gmail.com

b. Fisioterapeuta. angello_rocha@hotmail.com

c. Fisioterapeuta. Especialista em Saúde Pública pela Faculdade Nobre. rnfisio@yahoo.com.br
} 


\title{
PHYSIOTHERAPY AND ITS PRAXIS IN PRIMARY CARE \\ - a study from the perspective of students and teachers in the area of health at a university public in bahia.
}

\begin{abstract}
The present study aimed to identify the of undergraduate and faculty members perceptions of integration and Physiotherapist praxis in primary care in the area of health a University in Bahia. Methodology: A qualitative approach presents a socio-historical perspective, in which ten individuals were interviewed using semi structured interviews were divided into two groups, of which group I was composed of students, by group II teachers. The data were generated from the content analysis. According to the research participants the presence of the therapist in the family health strategy is required, but we could observe a blurring of the work object of this person, especially when you need interdisciplinary action, and culturally a rehabilitative practice be established. Thus, it is important to have an immediate change in vocational education of physiotherapists, greater awareness of the role and importance of this professionals in primary care research in addition to promoting the debate involving the need of the user.
\end{abstract}

Keywords: Primary Health Care; Physical Therapy Specialty; Human resources.

\section{INTRODUÇÃ̃O}

A atenção primária forma a base do sistema de saúde e deve ser o orientador de todo o processo de trabalho dos outros níveis de saúde, promovendo a organização das práticas e da distribuição dos recursos, tanto básicos como especializados, direcionados para a promoção, manutenção e meIhoria da saúde. Assim, à atenção primária a saúde (APS) é compreendida como parte do sistema que estabelece como porta de entrada do usuário para todas as necessidades e problemas, fornecendo atenção sobre a pessoa (não direcionada para a enfermidade) no decorrer do tempo e para todas as condições. ${ }^{(1)}$

Tal nível de atenção, representa o alicerce para que o sistema de saúde se solidifique, tornando-se mais eficaz, fortalecendo os vínculos entre os serviços de saúde e a população e contribuindo para a universalização do acesso e a garantia da integralidade e equidade da atenção, por isto tem como prioridade as populações com risco aumentado tanto do ponto de vista biológico como socioeconômico, no qual a saúde da família se apresenta como principal estratégia para a consolidação da Atenção Básica na rede de saúde brasileira. ${ }^{(2)}$

Destarte, a estratégia de saúde da família (ESF) propicia o enfrentamento e a busca pela resolução dos problemas de saúde identificados na comunidade cadastrada e desenvolve seu trabalho com uma equipe multiprofissional. A experiência em equipe possibilita a interdisciplinaridade permitindo aos profissionais a oportunidade de conviver de maneira mais próxima com a diversidade de conhecimentos e práticas, havendo um espaço para a troca de saberes. Nesse sentido, a ESF tem por objetivo ser o principal dispositivo de reorganização da atenção à saúde, entendendo a família como objeto principal e sua interação com o ambiente onde vive. ${ }^{(3)}$

Dentre as iniciativas do revisionismo obtido com a introdução da ESF, a APS ampliou seu leque 
de abrangência e se tornou um conjunto complexo de conhecimentos e procedimentos de uma intervenção ampla em diversos níveis sobre a qualidade de vida da população. ${ }^{(2)}$

No Brasil, a ESF caracteriza-se como ferramenta prioritária de um sistema de saúde constitucionalmente fundado no direito à saúde e na equidade do cuidado e, além disso, hierarquizado e regionalizado, como é o caso do Sistema Único de Saúde (SUS). Esta estratégia vem provocando um importante movimento e constante na tentativa de reorientação do modelo de atenção à saúde em nosso país. (3)

Para o fortalecimento da Atenção Básica, através da promoção da saúde, surgiu o Núcleo de Apoio à Saúde da Família - NASF. Tal núcleo foi instituído pelo Ministério da Saúde, em janeiro de 2008, com o objetivo de ampliar a abrangência das ações de Atenção Básica e melhorar a qualidade e a resolutividade da atenção à saúde. O mesmo são constituídos por equipes com diversos profissionais que desenvolvem suas ações em conjunto com os profissionais das ESF e são definidos pelos gestores municipais e pelas equipes de saúde da família a partir das necessidades locais, proporcionando a inserção de outros profissionais de saúde na ESF como os fisioterapeutas. $(4,5,6,7)$

Dessa forma foram necessárias diversas transformações em relação a atuação do Fisioterapeuta na Atenção Básica, entre as principais modificações, cita-se a apresentação de objetivos e ações voltadas para a prevenção e manutenção da saúde como principal fator de importância e não direcionado apenas à reabilitação. ${ }^{\left({ }^{(8)}\right.}$ É dentro desta nova perspectiva de atuação profissional que se insere o fisioterapeuta, agindo em programas de promoção de saúde e proteção específica em especial de saúde coletiva, focado em equipe multiprofissional abordando como eixos principais a universalidade, equidade, integralidade e a saúde vista em sua positividade. ${ }^{(9,10)}$

Desta forma, o presente estudo tem como objetivo conhecer a percepção dos discentes e docentes da área de saúde de uma Universidade na Bahia sobre a inserção e a práxis do fisioterapeuta na Atenção Básica.

\section{METODOLOGIA}

Este trabalho foi desenvolvido por uma abordagem qualitativa, numa perspectiva histórico- social que tem por objetivo traduzir e expressar o sentido dos fenômenos do mundo social; trata-se de reduzir a distância entre indicador e indicado, entre teoria e dados, entre contexto e ação.(11) O estudo foi realizado em uma Instituição Pública (Universidade Estadual do Sudoeste da Bahia), na qual a mesma é multi campi e o curso de Fisioterapia é oferecido no município de Jequié-Bahia localizda a $360 \mathrm{~km}$ da capital deste Estado.

O número de participantes da pesquisa ocorreu de acordo com a saturação das respostas na entrevista, totalizando uma amostra de dez participantes de forma intencional, sendo estes, três discentes de Fisioterapia, três discentes de outros cursos da área de saúde, dois docentes da área de saúde exceto do curso de Fisioterapia e dois docentes do curso de Fisioterapia. Os participantes da pesquisa foram divididos em Grupo I (Discentes - 6 participantes) e Grupo II (Docentes - 4 participantes), no qual teve o intuito de separar os discentes dos docentes da Instituição, em que poderá facilitar o entendimento a partir das suas devidas atribuições na Instituição. Como critério de inclusão para a pesquisa tivemos: indivíduos que estavam devidamente matriculados no curso de Fisioterapia e em outros cursos da área de saúde (Odontologia, Enfermagem e Medicina) da UESB (Universidade Estadual da Bahia e docentes da mesma instituição, e como critério de exclusão, indivíduos menores de idade.

A entrevista semiestruturada foi a técnica metodológica utilizada no presente estudo, seguindo um roteiro de entrevista com perguntas em relação a inserção do Fisioterapeuta na Atenção Básica e a sua Práxis neste nível de atenção, em especil, na Estratégia Saúde da Família, sendo a mesma realizada em abril de 2013. Tal método tem como carac- 
terística questionamentos básicos que são apoiados em teorias e hipóteses que se relacionam ao tema da pesquisa. Os questionamentos dão frutos a novas hipóteses surgidas a partir das respostas dos sujeitos da pesquisa. ${ }^{(12,13)}$

Os participantes da pesquisa foram informados em relação aos objetivos da pesquisa e normas éticas, assinando um termo de consentimento livre e esclarecido (TCLE). Durante as entrevistas, todas as perguntas e respostas foram gravadas.

Após leitura completa do material transcrito, releitura e organização das gravações, os dados das entrevistas foram ordenados através de um mapeamento de todas as informações obtidas, posteriormente foi realizada a classificação dos dados, onde foram identificados os núcleos de sentidos, que foram responsáveis pela criação de categorias empíricas (Necessidades da população/acesso, Inserção do fisioterapeuta na ESF, Atuação e formação acadêmica do fisioterapeuta). Por fim, os dados obtidos foram interpretados a partir do confronto entre o empirismo dos entrevistados com o referencial teórico da pesquisa buscando identificar as convergências e divergências entre os mesmos.

O presente estudo respeitou o que se preconiza a Resolução 196/96 do Conselho Nacional de saúde sobre as Diretrizes e Normas Regulamentadoras de Pesquisa envolvendo seres humanos, e foi realizado após a sua aprovação pelo Comitê de Ética em Pesquisa da Universidade Estadual do Sudoeste da Bahia (CEP-UESB), sob o número de protocolo $125.815 / 12$.

\section{ANÁLISE E DISCUSSÃO DOS RESULTADOS}

\section{DIFICULDADE DE INSERÇÃO DO FISIOTERAPEUTA NA ESF}

Um dos fatores apontados pelos entrevistados para que haja a inserção do fisioterapeuta na Atenção Básica é a falta de vontade política dos governan- tes, que em uma visão reducionista na gestão dos serviços públicos, compõem as equipes de saúde deste nível de Atenção com o número mínimo de trabalhadores, assim, aparece a dificuldade de inserção no contexto da entrevista, que foi descrita nas entrevistas abaixo:

A dificuldade acho que é o governo, dinheiro, custos, mobilizar isso aí, verbas, porque é necessário, tá na cara que é necessário o profissional de Fisioterapia na Atenção Básica (Ent 1- Fisioterapia; Grupo I).

\begin{abstract}
Acho que isso depende muito de vontade politica, a gente sabe que na Atenção Básica hoje apesar de ser recomendado não é preconizado que exista um fisioterapeuta na equipe mínima, mas acho que começa por aí, pelas próprias portarias, a politica nacional de saúde, mas acho que quando - município quer, a gestão se interessa isso acontece, acho que o principal é mais a questão política (Ent 4 - Odontologia; Grupo II).
\end{abstract}

Os gestores devem buscar atender às necessidades de saúde da população, da gestão setorial e do controle social em saúde, redimensionando o desenvolvimento da autonomia das pessoas até a condição de influência na formulação de políticas do cuidado. ${ }^{(14)}$

No estudde conclusãoi de curso realizado por Mello(15) os entrevistados intitulam a gestão municipal como principal responsável pelas políticas públicas para a Atenção Básica no município, incluindo a implantação dos Núcleos de Apoio a Saúde da Família (NASF) e a inserção do profissional fisioterapeuta na ESF. Os entrevistados percebem a necessidade de implantação de políticas públicas de saúde, mas que devido a gestão do município estudado não são realizadas. Portanto a universidade um papel fundamental no processo de esclarecimento da população, instigando a mesma a reivindicar seus direitos como cidadãos. Esta Estratégia permite a busca de ações que contemplem melhoria da atenção à saúde junto à gestão municipal.

Outro aspecto que, segundo os esntrevistados, interfere diretamente na inserção desse profissio- 
nal na Atenção Básica é a falta de definição do real objeto de trabalho do fisioterapeuta, sobre os seus limites e possibilidades, que ainda é um processo em construção. $O$ que foi apontado pelos entrevistados nas seguintes falas:

[...] a Fisioterapia tem que definir de fato o que vai fazer na Atenção Básica, carece ainda de uma definição quanto ao objeto de trabalho do fisioterapeuta na Atenção Básica, para que as outras profissões comecem a conhecer a importância do fisioterapeuta na Atenção Básica [...] o estudante tem que sair da graduação sabendo exatamente qual o papel dele na Atenção Básica pra que depois os outros profissionais venham a reconhecer essa necessidade [...] o próprio fisioterapeuta ainda não mostrou sua cara, não definiu seu papel ali na Atenção Básica (Ent 2 - Fisioterapia; Grupo I).

Uma das dificuldades que a gente tem para a inserção de uma forma mais efetiva na Atenção Básica é uma definição mais clara para os profissionais do que fazer na Atenção Básica, então começa com essa dificuldade que nos mesmos, os próprios profissionais que estão formando os alunos muitas vezes não temos uma noção clara do papel do profissional dentro do serviço, então esse seria o primeiro aspecto que talvez seja mais teórico do que prático e que seria um dos elementos que causam dificuldade para essa inserção (Ent 2 - Fisioterapia; Grupo II).

Para tal inserção na Atenção Básica é preciso uma atuação capaz de transformar as necessidades coletivas, e dessa forma a Fisioterapia precisa redimensionar seu objeto de intervenção, que deve estar ligado diretamente ao campo da promoção da saúde e da saúde coletiva sem abandonar suas competências ligadas à reabilitação. ${ }^{(16)}$

Vale ressaltar que além de não estar bem definido qual é o objeto de trabalho, outra questão fundamental é a atividade do fisioterapeuta associada com a equipe da Atenção Básica. No qual deve ser estabelecido de forma interdisciplinar com proposta de construção de projetos terapêuticos singulares, que possibilitem a potencialização da transformação na produção do cuidado junto à população produzindo vínculos $(5,14,17,18,19,20)$ a fala a seguir relata sobre esta condição:

\begin{abstract}
A possibilidade de melhora é quando conseguir realmente ter uma interdisciplinaridade, conseguir fazer com que os profissionais de saúde escutem uns aos outros, respeitem e entendam a importância de cada um na Atenção Básica e criar estratégias conjuntas (Ent 4 - Odontologia; Grupo II).
\end{abstract}

\section{ATUAÇÃO DO FISIOTERAPEUTA NA ESTRATÉGIA DE SAÚDE DA FAMÍLIA}

Desde que foi instituída a ESF, destaca-se que, ao contrário do modelo tradicional, centrado na doença e no hospital, a ESF tem como princípio norteador a priorização de ações que contemplem a proteção e promoção à saúde dos indivíduos e da família. ${ }^{(17)}$

Ribeiro $^{(8)}$ no entendimento da práxis idealiza e reforça a ideia para com os profissionais de saúde, observando que o ser humano quando visto apenas como um quadro clínico tem sua dimensão humana negada no que concerne principalmente torna-se diabético ou hipertenso, tratado de forma pejorativa, no qual a pessoa na verdade se encontra com a diabetes e a hipertensão.

Porém, a concepção do processo saúde / doença vem se transformando nas últimas décadas, principalmente no tocante ao modelo tecno-assistencial adotado, passando de um modelo hospitalocêntrico, curativo e reabilitador, para uma dimensão que compõe a promoção de saúde e prevenção de doenças como prioridades. Nessa perspectiva que a ESF se baseia entendendo a saúde não como o avesso da doença, mas como a busca do equilíbrio do ser humano, devendo, portanto, romper os estreitos limites da assistência curativa, havendo uma necessidade de atuação interdsciplinar para ampliar o conceito e consequentemente as necessidades de saúde. ${ }^{(17)}$

Diante do exposto os entrevistados intitulam a promoção à saúde como uma das principais atividades a serem realizadas pelo fisioterapeuta na 
Atenção Básica junto a uma equipe interdisciplinar, o que poderá redimensionar a resolutividade além do conhecimento dos trabalhadores que atuam na neste nível de Atenção, o que pôde-se perceber nas seguintes entrevistas:

[...] outras praticas que o fisioterapeuta pode realizar na Atenção Básica como por exemplo a formação, o ensino de cuidadores de pessoas idosas, além de questões relacionadas a ergonomia do lar e do trabalho daquela população, grupos de prevenção, palestras e inúmeras outras atividades (Ent 2 - Fisioterapia; Grupo I).

Juntamente com os outros profissionais ele estaria elaborando planos também pra tá pegando essa comunidade e vendo quais os problemas recorrentes [...] se essa população tem tendência a ser hipertensa com o hiperdia, se a população tem tendência a ter quedas, então que tipo de orientação a gente pode está fazendo dentro da Fisioterapia pra está prevenindo isso [...] por exemplo, usar-se um indicador de fragilidade nos idosos e com os resultados a gente poderia propor um programa pra melhorar a força e o equilíbrio desses idosos, seria uma coisa que nenhuma área faz atualmente e que a gente poderia está atuando (Ent 4 - Odontologia; Grupo I).

o fisioterapeuta na Atenção Básica deve atuar ligada a comunidade, orientando a comunidade sobre patologias futuras que ela possa ter relacionado a posicionamento, de como se deve varrer a casa e essas coisas assim, pra tentar evitar futuras alterações biomecânicas (Ent 5 - Enfermagem; Grupo I).

[...] ele 9 Fisioterapeuta) pode está trabalhando a educação em saúde, pensando principalmente na manutenção das capacidades funcionais porque ele vai lidar com todas as idades. Toda a parte de orientação, de educação em saúde do sistema osteomuscular principalmente e no funcionamento geral de todo o corpo. Por exemplo a questão postural, quantas vezes a gente tem problemas de coluna, problemas do aparelho locomotor em função de uma falta de orientação, se a gente tivesse uma orientação em saúde voltada pra isso a gente preveniria vários problemas do aparelho locomotor (ENT 10-Grupo II).
De acordo com as falas acima, nota-se que os entrevistados consideram a educação em saúde como ferramenta fundamental para atuação do fisioterapeuta na Atenção Básica, seja em condições em que o conhecimento é voltado especificamente (postura) para a Fisioterapia ou em questões gerais que envolvem outros profissionais (Hiperdia). É importante ressaltar que, o campo da intercisciplinaridade pode contribuir para uma ação integral para a produção do cuidado, mesmo em áres em que outros profissionais atuam

No entanto, exemplos de orientação de cuidadores de pessoas idosas; ergonomia do lar e do trabaIho; e associação de um diagnóstico da comunidade; aparecem como dispositivos disparadores de ações que podem ser realizadas por este profissional com uma equipe interdisciplinar, favorecendo assim, a comunidade atendida.

No estudo de Ragasson, ${ }^{(21)}$ ficaram evidenciadas algumas das atribuições do fisioterapeuta na Atenção Básica em relação à promoção e educação e em saúde que vão além das citadas anteriormente.

Contudo, é importante ressaltar que, a base da Fisioterapia, que é a reabilitação, não pode ser sucumbida, pois para os entrevistados a mesma é indissociável, além das necessidades dos usuários em relação ás suas condições de saúde, muitas vezes acamados, sendo destacado nos comentários abaixo que elucidam esta condição:

Ele não pode se esquecer da questão da reabilitação que é ligada realmente à profissão do fisioterapeuta pelo menos eu vejo isso de uma forma indissociável (Ent 2 - Fisioterapia; Grupo I).

[...] pessoas que estejam precisando se reabilitar de algum acidente e que moram naquela comunidade e que precisem do atendimento do fisioterapeuta, porque tem pessoas que passam por cirurgias e etc e tem que ir pra alguma clinica, tem que se deslocar da sua comunidade, sendo que poderá ter um fisioterapeuta naquela unidade de saúde (Ent 6 - Medicina; Grupo I). 
[...] assistência fisioterapêutica de pacientes que se encontram acamados, pacientes diabéticos, pacientes que demandam necessidades de serviços de Fisioterapia desde a área dermatológica até a área respiratória, neurológica, enfim as diversas áreas que comportam a Fisioterapia e que a gente poderia ta prestando essa assistência, seja no domicilio do próprio paciente, ou na unidade básica de saúde (Ent 2 - Fisioterapia; Grupo II).

Pode-se observar que a reabilitação permanece um campo de atuação segundo os entrevistados, principalmente na atenção domiciliar, o que contribuiria para a execução de ações específicas nas pessoas que possuem uma limitação funciona.

Complementando sobre a atuação do fisioterapeuta, o entrevistado 2; Grupo II acrescentou a gestão em saúde como competência e habilidades deste profissional como outra possível atuação na Atenção Básica, que pode ser um instrumento fundamental na gestão, pois a interdisciplinaridade a partir das várias áreas de atuação podem contribuir para uma visão integral do estabelecimento, o que foi relatado na seguinte fala:

[...] no aspecto administrativo, de gestão que acho que todo profissional de saúde deve ter competências e habilidades pra lidar com a parte administrativa, com a parte de gestão de uma unidade, de um serviço ou até de um sistema como um todo, isso seria uma das vertentes, mas que seria também algo abrangente a todos outros profissionais de saúde (Ent 2 - Fisioterapia; Grupo II).

Desta forma, esta fala pode contribuir com o fortalecimento do que está estabelecido nas diretrizes curriculares nacionais dos cursos de graduação em Fisioterapia. No qual, fica exposto que tais profissionais devem estar aptos a tomar iniciativas, fazer o gerenciamento e administração tanto da força de trabalho, dos recursos físicos e materiais e de informação, da mesma forma que devem estar aptos a serem empreendedores, gestores, empregadores ou lideranças na equipe de saúde. ${ }^{(21)}$

Destarte, o entrevistado 3; Grupo II acrescenta ainda sobre o processo de trabalho já existente na unidade básica de saúde que deve ser concebida em sua atuação do fisioterapeuta, respeitando as demandas já existentes, e atuando em conjunto com toda a equipe,

O fisioterapeuta para atuar na Atenção Básica ele precisa se adequar as demandas que já existem dentro da estratégia de saúde da família, que são as ações programáticas, dentro das ações programáticas e o profissional deve tentar reconhecer qual contribuição ele pode dar em cada ação programática dentro da sua particularidade, pensando na perspectiva multiprofissional [...] acho que qualquer profissional que vai atuar na estratégia de saúde da família ele deve se preocupar de que já existe uma estrutura formada, você não vai pra lá montar clinica de Fisioterapia, existe uma estrutura montada, existe ações programáticas que já estão planejadas pelo ministério da saúde, onde o enfermeiro, o médico e o odontólogo atuam, então os profissionais que se inserirem na estratégia da saúde da família devem atuar dentro da perspectiva das ações programáticas (Ent 3 - Fisioterapia; Grupo II).

Coadunando com esta fala, estudos descrevem que, de fato uma necessidade fundamental de adequação à realidade, porém há de convir, que a inserção deste profissional em um campo de atuação poderá trazer novos conhecimentos e exacerbar novas ideias e discussões que incentivem a construção de espaços de trabalho diferenciados com novas ações nos serviços de saúde. ${ }^{(18,20)}$

Por conseguinte, nos momentos de discussão da educação permanente em saúde de caráter interdisciplinar poderar contribuir para acontecimentos de desterritorialização e reterritotrialização através de intercessões no campo da micropolítica. Desta forma há uma possibilidade de contribuição na potencialização no campo ético-político dos trabalhadores e consequentemente refletiria na produção do cuidado com os usuários. Assim, poderá facilitar na (re) orientanção da construção de projetos terapêuticos singulares, redimensionando a práxis fisioterapêutica, oportunizando uma nova concepção. ${ }^{(22,23)}$ 
No entanto, há uma necessidade de discutir as diversas maneiras em que o profissional está sendo formado, desde as metodologias aplicadas e qual o desejo das mesmas, ou seja, não adianta modificá-las se mantêm o olhar reabilitador, é necessário que de fato seja experenciado novas práxis. ${ }^{(20,23)}$

\section{CONSIDERAÇÕES FINAIS}

Diante do exposto, nota-se que tanto a inserção como a práxis do fisioterapeuta na Atenção Básica ainda é indefinido. Há ainda muitas dúvidas sobre esta área de atuação definida nas diretrizes curriculares e em plena expansão no mercado de trabalho na atualidade brasileira.

No entanto, faz-se necessário que ações de caráter interdisciplinar e que práticas que possibilitem a produção do cuidado estejam na realidade deste profissional, que deve ir além da reabilitação, com ações de educação em saúde e de educação permanente no seu cotidiano.

Para tanto, vale a pena resslatar que a discussão sobre a formação do profissionalé de fundamental importância. que necessita de uma reflexão do objeto final destes projetos pedagógicos. Desta forma, a partir destas reflexões, as Universidades possibilitem formar profissionais diferenciados, capazes de realizar transformações nas vidas dos usurários...

\section{REFERÊNCIAS}

1. Starfield B. Atenção primária: equilíbrio entre necessidades de saúde, serviços e tecnologia. $2^{\mathrm{a}}$ Ed. Brasília: Unesco: Ministério da Saúde; 2004.

2. Serrano MM. Promoção da Saúde: um novo Paradigma. In: Ministério da Saúde. Textos de promoção da saúde. Brasília: Ministério da Saúde; 2002.

3. Brasil. Ministério da Saúde. Portaria no 2488. Política Nacional da Atenção Básica. Brasília, DF; 2011.
4. Mângial EF, Lancman S. Núcleos de Apoio à Saúde da Família: integralidade e trabalho em equipe multiprofissional. Rev. Ter. Ocup. Univ. 2008; 19 (2):1.

5. Brasil. Ministério da Saúde. Diretrizes do Núcleo de Apoio a Saúde da Família. Brasília, DF: Ministério da Saúde: 2009. (Caderno de Atenção Básica, n. 27)

6. Nascimento MC, Sampaio RF, Salmela JH, Mancini MC, Figueiredo IM. A profissionalização da Fisioterapia em Minas Gerais. Rev bras fisioter. 2006; 10(2): 241-247.

7. Souza MC, Bomfim AS, Souza JN, Franco TB. Fisioterapia e Núcleo de Apoio à Saúde da Família: conhecimento, ferramentas e desafios. Mundo da Saúde. 2013; 37(2): 176-184.

8. Ribeiro K. A atuação da fisioterapia na atenção primária à saúde. Fisioter Brasil; 2OO2, 3(5):2), $11-318$.

9. Conill EM. Políticas de atenção primária e reformas sanitárias: discutindo a avaliação a partir da análise do Programa Saúde da Família em Florianópolis, Santa Catarina, Brasil, 1994-2000. Cad. Saúde Pública. 2002; 18(Suplemento):191-2O2.

10. Deliberato P. Fisioterapia preventiva. São Paulo: Manole; 2002.

11. Minayo MCS. O desafio do conhecimento. $8^{\text {a }}$ Ed. São Paulo: Hucitec; 2004.

12. Triviños ANS. Introdução à pesquisa em ciências sociais: a pesquisa qualitativa em educação. São Paulo: Atlas; 2009.

13. Gil AC. Métodos e técnicas de pesquisa social. 6. ed. São Paulo, Atlas, 2008.

14. Bonfim LA, Bastos ACS. O Impacto sóciocultural do Programa de Saúde da Família (PSF): uma proposta de avaliação. Cad saúde publica 1998; 14(2):429-435. Tradução Leny Alves Bonfim.

15. RIBEIRO, K. S. Q. S.2 Melo R. Formação acadêmica do fisioterapeuta para a Atenção Básica 26 fls. [dissertação]. Feira de Santana: Universidade Estadual do Sudoeste da Bahia; 2011.

16. Bispo Júnior JP. Formação em Fisioterapia no Brasil: reflexões sobre a expansão do ensino 
e os modelos de formação. Hist ciênc saúdeManguinhos. 2009;16 (3):655-68RIBEIRO, K. S. Q. S.2.

17. Souza MC, Souza JN. Saúde coletiva: um campo de diversos saberes e diversos olhares. Vitória da Conquista: Edições UESB; 2013.

18. Souza MC, Araújo TM, Reis Júnior WM, Souza JN, Vilela ABA, Franco TB. Integralidade na atenção à saúde: um olhar da equipe de Saúde da Família sobre a Fisioterapia. O mundo da saúd. 2012; 36 (3): 452- 460.

19. França AJ, Andrade FA, Araújo TM, Souza MC. Relação intraequipe e produção de vínculos em uma Unidade de Saúde da Família. Efdeportes, 2014; 18 (190): 1-6.

2O. Souza MC, Bomfim AS, Souza JN, Franco TB. Fisioterapia e Núcleo de Apoio à Saúde da Família: conhecimento, ferramentas e desafios. O mundo da saúde. 2013; 37 (2): 176-184.
21. Ragasson CAP, Almeida DCS, Comparin K, Mischiati MF, Gomes JT. Atribuições do fisioterapeuta no Programa de Saúde da Família: reflexões a partir da prática profissional; 2003. Disponível em: www. unioeste.br/projetos/saudefamilia/ atribuicoes_ psf.rtf.

22. Merhy EE. Integralidade: implicações em xeque. In: Pinheiro R, Ferla AA, Mattos RA, organizadores. Gestão em redes: tecendo os fios da integralidade. Rio de Janeiro; 2006.

23. Feverwerker LCM. Micropolítica e saúde: produção do cuidado, gestão e formação. 1. ed. Porto Alegre: Editora Rede Unida; 2014 